\title{
Consequences of neutrinoless double decays dominated by short ranged interactions
}

\author{
C. Q. Geng ${ }^{1,2,3,4, *}$ and John N. Ng ${ }^{5, \dagger}$ \\ ${ }^{1}$ School of Fundamental Physics and Mathematical Sciences, Hangzhou Institute for Advanced Study, \\ UCAS, Hangzhou 310024, China \\ ${ }^{2}$ International Centre for Theoretical Physics Asia-Pacific, Beijing/Hangzhou, China \\ ${ }^{3}$ Department of Physics, National Tsing Hua University, Hsinchu 300, Taiwan \\ ${ }^{4}$ National Center for Theoretical Sciences, Hsinchu 300, Taiwan \\ ${ }^{5}$ TRIUMF, 4004 Wesbrook Mall, Vancouver, British Columbia V6T 2A3, Canada
}

(Received 22 March 2020; accepted 30 June 2020; published 16 July 2020)

\begin{abstract}
We investigate some consequences if neutrinoless double beta decays $(0 \nu \beta \beta \mathrm{s})$ of nuclei are dominated by short range interactions. To illustrate our results, we assume that $0 \nu \beta \beta$ s proceed mainly through short range interactions involving two-W-boson exchanges and confine ourselves to only include new scalars without new gauge interactions for the SM fermions. For the neutrino mass problem, we propose to solve it by adopting that the light neutrinos have predominantly Dirac masses and the small Majorana masses induced by the new scalars render them quasi-Dirac particles. This particular aspect of neutrinos may be detectable in the next generations of neutrino oscillation experiments and/or neutrino telescope. If so this opens a new connection between $0 \nu \beta \beta$ and neutrino physics. We also note the new physics signals such as the high charged states that can be explored in hadron colliders. In particular, we find that a high energy $e^{-} e^{-}$will be very useful in testing the origin of lepton number violation, which complements the $0 \nu \beta \beta$ studies.
\end{abstract}

DOI: $10.1103 /$ PhysRevD.102.013004

\section{INTRODUCTION}

In this paper we investigate the possibility that neutrinoless double beta decays $(0 \nu \beta \beta \mathrm{s})$ of nuclei are dominated by short range physics not involving a heavy sterile righthanded neutrino but due to some other new physics beyond the Standard Model (SM). This is in sharp contrast to the usual assumption that $0 \nu \beta \beta$ is due to the exchanges of light Majorana neutrinos, which constitute a long range exchange force between decaying nucleons in the nucleus. The half-life of the decaying nucleus is directly proportional to the masses of the exchange or virtual neutrinos. They are identified as the active neutrinos of the SM, which are known to be massive but light, i.e., less than $1 \mathrm{eV}$, due to the observed neutrino oscillations. We refer this as the three-Majorana-neutrino paradigm (3MNP). This is an economical and elegant framework for $0 \nu \beta \beta$ as it involves only physics in the SM with the violation of lepton number encoded in the Majorana masses of the active neutrinos.

\footnotetext{
*geng@phys.nthu.edu.tw misery@triumf.ca
}

Published by the American Physical Society under the terms of the Creative Commons Attribution 4.0 International license. Further distribution of this work must maintain attribution to the author(s) and the published article's title, journal citation, and DOI. Funded by SCOAP ${ }^{3}$.
For an up to date review, see $[1,2]$. However, since the origin of the active neutrinos masses and their nature is an open question, it behooves us to examine alternatives to the above paradigm and to study consequences that they will lead to, in particular to search for new pathways that are not evident in the 3MNP.

The scenario we are interested in assumes that $0 \nu \beta \beta \mathrm{s}$ proceed predominately by short range physics beyond the SM. The long range potential due to the light Majorana neutrino exchange plays either a negligible or subdominant role. Admittedly, this is an ad hoc assumption. This can happen in several ways. First, if all the active neutrinos are Majorana particles, their mixings and mass squared differences are given by oscillations experiments. If their masses are ordered as in the normal hierarchy with the lightest mass being small, then they will induce $0 \nu \beta \beta \mathrm{s}$ at a rate well below the sensitivity of near future experiments, see, e.g., [1,2]. A less stringent possibility is that the Majorana phases are such that they cancel in the effective $\nu_{e}$ Majorana mass. This amounts to that the first element $M_{\nu}$ of the active neutrino mass matrix in the weak basis is vanishing, i.e., $\left(m_{L}\right)_{e e} \simeq 0$. A third possibility is that the three active neutrinos participating in oscillations have dominantly Dirac masses. By that we mean their masses are given by the usual Higgs mechanism via the Yukawa couplings terms $y v \bar{N} \nu$, where $N$ denotes a SM singlet neutral lepton and other notations are obvious. More details 
are given in Sec. III. Oscillations data cannot distinguish whether the active neutrinos are Dirac or Majorana in nature. If any of the above scenarios takes place and $0 \nu \beta \beta$ is observed in the next generations of experiments then it is likely that some new short range physics is operative. Short range physics contributions to $0 \nu \beta \beta$ s have been discussed in $[3,4]$ concentrating in how they affect the hadronic physics. They have been parametrized by effective operators of dimension 7 and $9[5,6]$. A more recent study of short range physics in $0 \nu \beta \beta$ is given in [7], which used the effective operator approach. Since dimension 7 operators will involve a light neutrino exchange, they will fall out of our assumptions. That leaves dimension 9 as the lowest dimensional operator we need to consider.

The theory space for new physics that can generate these operators is large. To reduce that we make a conservative assumption that all SM fermions do not carry additional quantum numbers than dictated by the SM gauge symmetry. This is supported by LHC having not seen any new gauge bosons and numerous low energy precision measurements that set stringent limits on their masses and couplings. This leaves new scalars and fermions transform nontrivially under the SM gauge symmetry that can carry color and color singlets as new degrees of freedom to be studied. Here, we shall concentrate on scalars and leave new fermions for a future work.

As we noted before, short range interactions have been discussed previously. Our approach is similar to that of [7] (AABSW) in that we both relate $0 \nu \beta \beta$ to the Majorana mass generation for active neutrinos. AABSW uses exclusively effective operators and is very general, whereas we concentrate on $2-\mathrm{W}$ exchange and construct UV complete models. This allows us to give a more quantitative estimate of the active neutrino Majorana masses and conclude that they are too small to accommodate oscillation data and led us to the considerations of pseudo-Dirac oscillations. We also limit to no new symmetries added to the SM. Left-right symmetry in $0 \nu \beta \beta$ was studied in [8], while R-parity violation supersymmetry was explored in [9].

We organize our paper as follows. In Sec. II, we take two-W-boson exchange as the lowest state for the dimension 9 operator. Then new physics for $0 \nu \beta \beta$ will proceed via $W W \rightarrow e e$. Tree level new physics for this will involve colorless scalars. We then compare the constraints given by $0 \nu \beta \beta$ with that from the LHC and future colliders on these new objects. Since the interactions involve must violate lepton number by 2 units, one has to check that they do not generate $\left(m_{L}\right)_{e e}$ at a large enough value so as to invalid our short range dominance proposition. Although conceptually similar, this is independent of the black box theorem [10], which generates a Majorana mass for $\nu_{e}$ at the 4-loop level. This yields $\left(m_{L}\right)_{e e} \lesssim 10^{-28} \mathrm{eV}$ [11] without the need to specify what leads to $0 \nu \beta \beta$. This value is inconsequential for $0 \nu \beta \beta$ if they were to be discovered in the current or next generation experiments. In Sec. III, we investigate the issue of neutrino masses if $0 \nu \beta \beta$ is driven by the short range interactions proposed. In Sec. IV, we discuss collider signatures for some high charge states. Our conclusions are given in Sec. V.

\section{2-W-BOSONS MECHANISM FOR $0 \nu \beta \beta$}

At the quark level, $0 \nu \beta \beta$ can be represented by Fig. 1 with the 2-W-boson mechanism being the leading approximation as depicted.

The Lagrangian for the short range interaction can be symbolically written as

$$
\mathcal{L}=\frac{G_{F}^{2}}{2 m_{p}} \sum_{i} \epsilon_{i} J_{i} J_{i} j_{i}
$$

where $i$ denotes different electron currents $\left(j_{i}\right)$ and quark currents $\left(J_{i}\right), \epsilon_{i}$ represents the particle physics involved, and $m_{p}$ stands for the proton mass. Here, Lorentz contractions have to be taken and are not shown, while the quark currents are to be sandwiched between initial and final nuclear states for the full matrix element. The half-life for a given decay may be generically expressed as

$$
T_{\frac{1}{2}}^{-1}=\left|\epsilon_{i}\right|^{2} G_{i}\left|\mathcal{M}_{i}\right|^{2}
$$

where $G_{i}$ is the nuclear phase space factor, and $\mathcal{M}_{i}$. The calculations of these two factors are given in [3]. In this paper, we are concerned with models that give rise to $\epsilon_{i}$ and it is dimensionless. As a comparison, the long range neutrino exchange is given by $\epsilon_{\nu}=\left(m_{L}\right)_{e e} /(0.01 \mathrm{eV})$.

For the 2-W-boson scattering mechanism one can construct tree level new physics that induces $W^{-*}+$ $W^{-*} \rightarrow e^{-}+e^{-}$. Immediately one can recognize that a doubly charged scalar $T^{--}$will be involved. A generic $T^{--}$ exchange mechanism is displayed in Fig. 2. The nature of $T^{--}$depends on whether the chirality of the final state electrons. A detailed examination of this is given next.
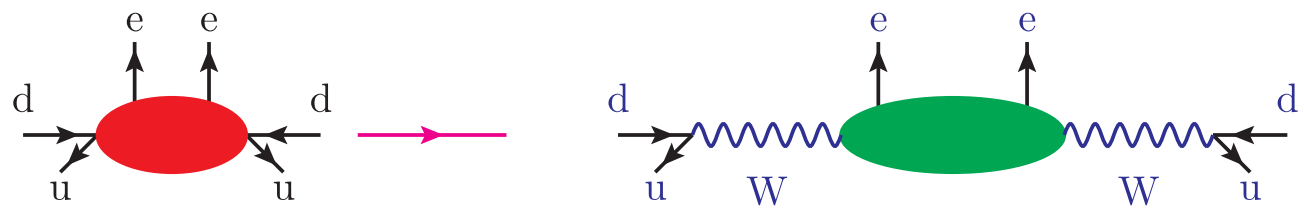

FIG. 1. Feynman diagrams for the short range interaction of neutrinoless double beta decays, where the right-hand side indicates the 2-W-boson exchange mechanism. 


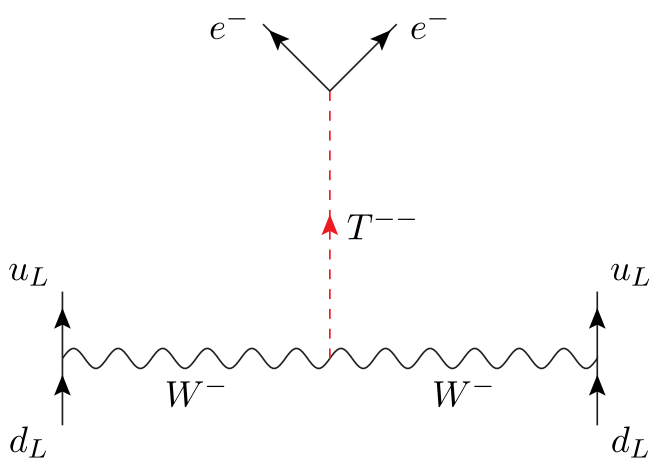

FIG. 2. Generic doubly charged scalar exchange for neutrinoless double beta decays.

\section{A. $W^{-*}+W^{-*} \rightarrow e_{L}+e_{L}$}

If the electrons are left-handed they are in a SM doublet with the active neutrinos written as $\ell=\left(e_{L} \nu_{L}\right)$. Then $T$ will be in a $S U(2)_{L}$ triplet with hypercharge $Y=1$ where we use the normalization $Q=T_{3}+Y$ with standard notations. Explicitly, $T$ consists of three states: $\left(T^{++}\right.$, $\left.T^{+}, T^{0}\right)$. A coupling between $T$ and the lepton, given by $y_{\ell} \overline{\ell^{c}} T \ell$, can be constructed. This type of Higgs triplet models is popular in type II and radiative seesaw models for neutrino masses, see e.g., [12,13]. The $T^{0}$ component must pick up a VEV, $v_{T}$, in order to get a $W^{-} W^{-} T^{++}$ coupling. This in turn generates a tree level neutrino Majorana mass; hence $y_{\ell} v_{T}<1 \mathrm{eV}$. Thus, the $W W T$ coupling is $\sim g v_{T}$, where $g$ is the $S U(2)$ gauge coupling. Since no doubly charged scalar has been found at the LHC $[14,15]$, a lower bound on the mass is $1.3 \mathrm{TeV}$ by using the signal of same sign dileptons and assuming $100 \%$ branching ratio. The rate for $0 \nu \beta \beta$ is given by $g y_{\ell} v_{T} m_{p} / M_{T}^{2}$. We estimate that $\epsilon \lesssim 10^{-24}$ and so this case is uninteresting. We conclude that triplet Higgs with SM quantum numbers $(1,3,1)$ in usual notations will not be useful for us.

\section{B. $W^{-*}+W^{-*} \rightarrow e_{R}+e_{R}$}

The electron pairs are in a singlet state of $(1, \mathbf{1},-2)$ under $S U(3)_{C} \times S U(2)_{L} \times U(1)_{Y}$. A doubly charged scalar singlet $\Phi(1,1,2)$ can couple to them with the coupling $1 / 2 y_{\Phi} \overline{e_{R}^{c}} e_{R} \Phi$. Now $y_{\Phi}$ is unconstrained by neutrino masses. On the other hand, $\Phi$ will have no tree level coupling to the two W-bosons. This necessitates the introduction of additional Higgs scalars. We have previously ruled out the scalar $(1,3,1)$ and it is easy to see that triplets with $|Y| \geq 2$ cannot be used. This leaves the option of higher $S U(2)$ representations. The next lowest representation that can be used is $\Psi(1,4,3 / 2)$ and explicitly the quadruplet states are $\left(\psi^{+++}, \psi^{++} / \sqrt{3}, \psi^{+} / \sqrt{3}, \psi^{0}\right)$. If $\left\langle\psi^{0}\right\rangle=v_{\psi} \neq 0$ the vertex $W^{-} W^{-} \psi^{++}$can be generated and is $i g v_{\psi} / 2$. The hypercharge assignments also prevents tree level couplings to active neutrinos.

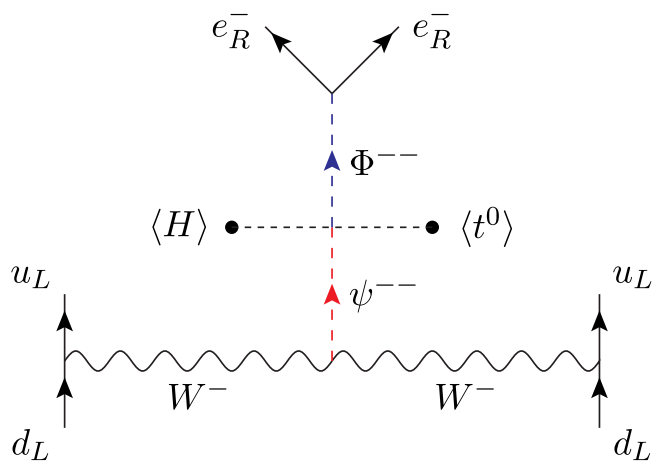

FIG. 3. Neutrinoless double beta decays via the doubly charged Higgs exchange in the weak interaction basis.

The next ingredient is to provide the mixing between $\Psi^{++}$and $\Phi^{++}$. The price to pay is to introduce yet another scalar $T^{\prime}(1,3,0)=\left(t^{-}, t^{0} / \sqrt{2}, t^{+}\right)$. Then the gauge invariant term $H T^{\prime} \Psi \Phi^{\dagger}$ is allowed. ${ }^{1}$ We obtain the desired mixing if $\left\langle t^{0}\right\rangle$ is nonvanishing and $\langle H\rangle=v$. We note that both $\left\langle t^{0}\right\rangle$ and $\left\langle\psi^{0}\right\rangle$ must be less than a $\mathrm{GeV}$ from precision electroweak measurements. Moreover, without exotic fermions the hypercharges of the new scalars are such that they have no tree level couplings to active neutrinos. Thus, $0 \nu \beta \beta$ is given by Fig. 3. Our solution is not unique and higher $S U(2)$ representations can be used. Constructing viable models can simply follow what we have presented.

We return to the discussion of our model. First, we can identify the origin of lepton number violation. It is the four scalar term $H T^{\prime} \Psi \Phi^{\dagger}$ after the SSB of the Higgs fields. A mixing of $\Phi$ and $\Psi$ is then generated. Explicitly, one has that

$$
\lambda H T^{\prime} \Psi \Phi \stackrel{\mathrm{SSB}}{\rightarrow} \frac{v_{T} v}{\sqrt{6}} \psi^{++} \Phi^{++},
$$

where $v$ is the SM Higgs VEV and $\lambda$ is a free parameter that controls the strength of lepton number breaking. The physical doubly charged scalars can be obtained by diagonalizing a $2 \times 2$ mass matrix with off diagonal terms given by Eq. (3). The details depend on the scalar potential and are not essential for this discussion. It suffices to know that the mixing angle $\alpha$ is given by

$$
\sin 2 \alpha \sim \frac{\lambda v_{T} v}{M_{\Psi}^{2}-M_{\Phi}^{2}}
$$

where $M_{\Psi}$ and $M_{\Phi}$ are the masses of the respective fields before diagonalization and we have omitted some unimportant constants. $v_{T}$ is constrained to be less than a few

\footnotetext{
${ }^{1}$ If economy on new states is desired one can construct a soft term such as $\Psi \Psi \Phi^{\dagger}$. Mixing between $\Psi$ and $\Phi$ is induced after the spontaneous symmetry breaking (SSB) of $\Psi$. However, this will require that $\Psi$ be in an odd dimensional $S U(2)$ representation. For our case the lowest one would be a quintuplet [16].
} 
$\mathrm{GeV}$, and the masses in the denominator are of order $0.5 \mathrm{TeV}$. This is the lower bound from [14] when the branching ratio of a doubly charged scalar into a given same sign dilepton pair is $10 \%$. This is more appropriate for us since we expect $\Phi \ell \ell^{\prime}$ to be approximately equal. This gives 9 such decays. If the mass splitting is also of order $100 \mathrm{GeV}$, we expect $\alpha \lesssim 10^{-3}$ if $\lambda \sim 1$. A larger mixing can occur if the masses are accidentally degenerate or $\lambda \sim 10$. The physical states denoted by $S_{1,2}^{ \pm \pm}$are related to the weak states $\Phi^{ \pm \pm}$and $\psi^{ \pm \pm}$via

$$
\begin{aligned}
& \Phi=\cos \alpha S_{1}+\sin \alpha S_{2}, \\
& \Psi=-\sin \alpha S_{1}+\cos \alpha S_{2} .
\end{aligned}
$$

Without lost of generality, we assume that $S_{1}$ is the lighter state. As we shall see later, the mixing is small and $S_{1}$ is mostly $\Phi$. The masses are denotes by $M_{1,2}$, respectively.

Referring to Eq. (1), our model gives only one contribution and the current correlation has the form $J^{\mu} J_{\mu} j$ where $J^{\mu}=\bar{u} \gamma^{\mu}\left(1-\gamma^{5}\right) d$ and $j=\bar{e}^{c}\left(1+\gamma^{5}\right) e$. Using $\bar{y}_{\Phi}=y_{\Phi} / g, \epsilon$ is given by

$$
|\epsilon| \sim m_{e} v_{\psi} \sin 2 \alpha \bar{y}_{\Phi}\left(\frac{1}{M_{1}^{2}}-\frac{1}{M_{2}^{2}}\right) .
$$

It is sensitive to the difference of the inverse mass squared $\Delta^{2}=1 / M_{1}^{2}-1 / M_{2}^{2}$. Currently, the half-life of the decay ${ }^{136} \mathrm{Xe} \rightarrow{ }^{136} \mathrm{Ba} e^{-} e^{-}[17]$ gives the most stringent limit [3] ${ }^{2}$

$$
\epsilon \lesssim 5 \times 10^{-9}
$$

Thus, we get

$$
\frac{y_{\Phi}}{g} \leq 1.25\left(\frac{.001}{\alpha}\right)\left(\frac{1 \mathrm{GeV}}{v_{\psi}}\right)\left(\frac{\sqrt{\Delta^{2}}}{0.5 \mathrm{TeV}}\right)^{2} .
$$

This shows the complementarity of $0 \nu \beta \beta$ to direct searches at the LHC. The direct search is sensitive to one state at a time and depends on the decay products of the state in question due to experimental constraints. If $y_{\Phi} / g \simeq 1$, it implies that the branching ratio of decay of the doubly charged scalar into same sign dilepton pair is not negligible compared to that into a pair of same sign W-bosons. This is in fact the preferred search mode at the LHC. This holds true if the mixing is small as we argue. However, if the scalars are more degenerate and the mixing becomes large, then the gauge bosons decays can become more important. This is more challenging experimentally but important to test the physics involved and must not be ignored.

\footnotetext{
${ }^{2}$ Other experiments include ${ }^{130} \mathrm{Te}$ [18] and ${ }^{76} \mathrm{Ge}$ [19] decays. They give a factor of 2 to 5 less stringent limit on $\epsilon$.
}

\section{NEUTRINO MASS GENERATION}

An examination of Fig. 3 will show that the lepton number violating interaction constructed will yield a 2-loop contribution to a Majorana mass to $\nu_{e}$. The Feynmann diagram is given in Fig. 4, where it is depicted in the weak basis. Evaluating the diagram [12] for a given physical scalar $S$ gives

$$
\begin{aligned}
\left(m_{L}\right)_{e e}= & g^{4} m_{e}^{2} v_{\psi} \overline{y_{\Phi}} \sin 2 \alpha\left[I\left(M_{W}^{2}, M_{1}^{2}, m_{e}^{2}\right)\right. \\
& \left.-I\left(M_{W}^{2}, M_{2}^{2}, m_{e}^{2}\right)\right] .
\end{aligned}
$$

The integral I is given by

$$
\begin{aligned}
I\left(M_{W}^{2}, M_{S}^{2}, m_{e}^{2}\right)= & \int \frac{d^{4} q}{(2 \pi)^{4}} \int \frac{d^{4} k}{(2 \pi)^{4}} \frac{1}{k^{2}-m_{e}^{2}} \\
& \times \frac{1}{k^{2}-M_{W}^{2}} \frac{1}{q^{2}-M_{W}^{2}} \\
& \times \frac{1}{q^{2}-m_{e}^{2}} \frac{1}{(k-q)^{2}-M_{s}^{2}} .
\end{aligned}
$$

A similar cancelation between $S_{1}$ and $S_{2}$ takes place as in $0 \nu \beta \beta$. Assuming $M_{2} \gg M_{1} \gg M_{W}$, one has that

$$
I\left(M_{W}^{2}, M_{1}^{2}, 0,0\right) \sim \frac{1}{(4 \pi)^{4}} \frac{1}{M_{1}^{2}} \ln ^{2}\left(\frac{M_{W}^{2}}{M_{1}^{2}}\right) .
$$

Comparing Eqs. (6), (9), and (11), we see that $\left(m_{L}\right)_{e e}$ is completely determined by the amplitude for $0 \nu \beta \beta$. There are three suppression factors at play. First, the mixing $\alpha$ is small, $10^{-3}$, second there is the 2-loop factor which is $\sim 10^{-5}$, and finally the smallness of $m_{e}$. The last one is the biggest suppression because $m_{e}^{2} / M_{S}^{2} \sim 10^{-12}\left(10^{-14}\right)$ for a $\mathrm{TeV}(10 \mathrm{TeV})$ scalar. Even with $v_{\psi} \sim 1 \mathrm{GeV}$, and $g^{4}=0.2$ we get $\left(m_{L}\right)_{e e} \lesssim 10^{-13}$ to $10^{-15} \mathrm{eV}$ for the two values of $M_{S}$. Hence, the active $\nu_{e}$ will give a negligible contribution to $0 \nu \beta \beta$ and is consistent with our short range physics dominance hypothesis. Nevertheless, we need to look deeper into what features of neutrino physics the model will predict. To this end it is sufficient to examine the flavor diagonal elements of the active neutrino mass it generates; i.e., the $\left(m_{L}\right)_{e e},\left(m_{L}\right)_{\mu \mu},\left(m_{L}\right)_{\tau \tau}$ entries. From Fig. 4 and Eq. (9), it is easy to see that the largest element of the $3 \times 3$ neutrino mass matrix is the $\tau \tau$ entry. We estimate that

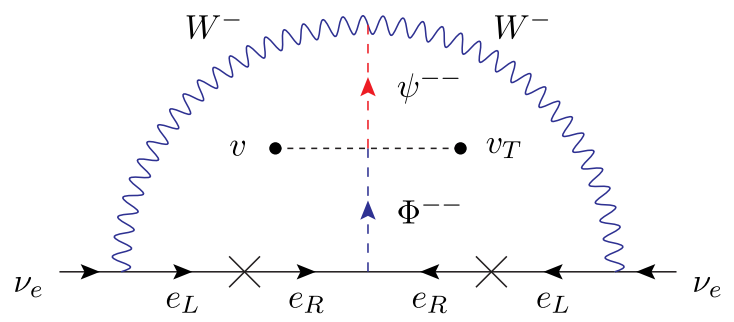

FIG. 4. 2-loop diagram for $\nu_{e}$ Majorana mass. 


$$
\left(m_{L}\right)_{\mu \mu}=\frac{m_{\mu}^{2}}{m_{e}^{2}}\left(m_{L}\right)_{e e}=4.3 \times 10^{4}\left(m_{L}\right)_{e e} \sim 10^{-9}\left(10^{-11}\right) \mathrm{eV}
$$

$\left(m_{L}\right)_{\tau \tau}=\frac{m_{\tau}^{2}}{m_{e}^{2}}\left(m_{L}\right)_{e e}=1.2 \times 10^{7}\left(m_{L}\right)_{e e} \sim 10^{-6}\left(10^{-8}\right) \mathrm{eV}$,

where we have assumed that the Yukawa couplings of $\Phi$ to $e, \mu, \tau$ are the same. Since the neutrinos oscillation data involve the mass squared differences of the neutrinos, it is obvious that we are many orders of magnitude away from explaining the data [20]. The smallest mass squared difference is $7.39 \times 10^{-5} \mathrm{eV}^{2}$. Pushing some Yukawa couplings to their perturbative limits will not change the above conclusion.

A solution to this conundrum will be to introduce $3 \mathrm{SM}$ singlet right-handed neutrinos $N_{R}$ that can be used to give Dirac masses to the active neutrinos. The physics here can be seen simply by considering the single family case with only $\nu_{e L}$ and only one $N_{R}$. Since we assume that lepton number violation occurs only in the scalar sector, we take $N_{R}$ to have a vanishing tree level Majorana mass. $\nu_{e}$ develops a relatively large Dirac mass via the usual Yukawa coupling of $y_{\nu} \bar{\nu}_{L} N_{R} H$. If $y_{\nu} \sim 10^{-12}$, then a Dirac mass $m_{D} \sim 0.1 \mathrm{eV}$ is generated for $\nu_{e}$ after the SSB. We treat all Yukawa couplings as free parameters and will not go into a deeper understanding of the hierarchy shown in known Yukawa couplings. Moreover, such a small Yukawa coupling can be implemented in extra dimensional models [21].

For typographic simplicity, we drop all subscripts for the lepton states in the following. The $2 \times 2$ mass matrix in the $\nu, N^{c}$ basis is represented by

$$
M_{\nu}=\left(\begin{array}{cc}
m_{e e} & m_{D} \\
m_{D} & M_{N}
\end{array}\right)
$$

Initially, $m_{e e}=0$ due to $S U(2)$, and we can set $M_{N}=0$ although ad hoc is nevertheless technically natural for theories below the weak scale with $N$ not integrated out. After the symmetry breaking, we obtain $m_{D} \gg m_{e e}$ as seen previously. Moreover, $M_{N}$ is induced at 3-loop and thus $m_{e e} \gg M_{N}$. Since this has a very small value comparing to even $m_{e e}$ we can safely set it to zero. The eigenvalues for Eq. (14) are

$$
\begin{aligned}
m_{ \pm} & \simeq m_{D}(1 \pm \delta), \\
\delta & =\frac{m_{e e}}{2 m_{D}},
\end{aligned}
$$

and the states are almost maximally mixed, i.e.,

$$
\begin{aligned}
& \nu_{+} \simeq \frac{1}{\sqrt{2}}\left[(1+\theta) \nu+(1-\theta) N^{c}\right], \\
& \nu_{-} \simeq \frac{i}{\sqrt{2}}\left[(-1+\theta) \nu+(1+\theta) N^{c}\right],
\end{aligned}
$$

with the small mixing given by $\theta=m_{e e} /\left(4 m_{D}\right)$. The mass eigenstates are a pair of Majorana leptons with opposite $C P$ phases with a very small mass splitting, which in our example is proportional to $m_{e e} \sim 10^{-11} \mathrm{eV}$. This is known as quasi or pseudo-Dirac neutrinos $[22,23] .^{3}$

Generalization to 3 families of neutrinos is straightforward but nontrivial. Firstly, three righthanded SM singlet neutrinos $N_{\alpha R}$ are introduced and denote their masses by the matrix $\left(\mathbf{m}_{\mathbf{R}}\right)_{\alpha}$, where $\alpha$ denotes weak eigenbasis. Explicitly, the weak eigenstates are

$$
\psi_{L}=\left(\begin{array}{c}
\nu_{\alpha L} \\
N_{\alpha R}^{c}
\end{array}\right), \quad(\alpha=e, \mu, \tau)
$$

where the superscript $c$ denotes charge conjugation. The neutrino mass matrix is now a $6 \times 6$ matrix denoted by

$$
M=\left(\begin{array}{cc}
\mathbf{m}_{\mathbf{L}} & \mathbf{m}_{\mathbf{D}} \\
\mathbf{m}_{\mathbf{D}}^{\dagger} & \mathbf{m}_{\mathbf{R}}
\end{array}\right),
$$

where each entry $\mathbf{m}$ is a $3 \times 3$ matrix. In our model $\left(\mathbf{m}_{\mathbf{L}}\right)_{\alpha \beta}$ can be obtained by calculating similar diagrams of Fig. 4 . $\mathbf{m}_{\mathbf{D}}$ is an obvious generalization of the Dirac mass and is the dominant matrix, i.e., all the elements are such that $m_{D} \gg m_{L} \gg m_{R} \simeq 0$. It is more convenient to diagonalize the product $M^{\dagger} M$, which reads as

$$
M^{\dagger} M \simeq\left(\begin{array}{ll}
\mathbf{m}_{\mathbf{D}}^{\dagger} \mathbf{m}_{\mathbf{D}} & \mathbf{m}_{\mathbf{L}}^{\dagger} \mathbf{m}_{\mathbf{D}} \\
\mathbf{m}_{\mathbf{D}}^{\dagger} \mathbf{m}_{\mathbf{L}} & \mathbf{m}_{\mathbf{D}}^{\dagger} \mathbf{m}_{\mathbf{D}}
\end{array}\right)
$$

where the dominance of $\mathbf{m}_{\mathbf{D}}$ has been employed. This can be diagonalized by [25]

$$
V=\frac{1}{\sqrt{2}}\left(\begin{array}{cc}
U & i U \\
U_{R} P & -i U_{R} P
\end{array}\right) .
$$

$U$ is the usual Pontecorvo-Maki-Nakagawa-Sakata matrix [26,27] and it renders $\mathbf{m}_{\mathbf{D}}{ }^{\dagger} \mathbf{m}_{\mathbf{D}}$ diagonal with the eigenvalues $m_{1}^{2}, m_{2}^{2}, m_{3}^{2}$. Defining $\left.\varepsilon_{i}=\left(U^{\dagger} \mathbf{m}_{\mathbf{L}} U\right)_{i i}\right)$ and $P$ a diagonal phase matrix $e^{i \phi_{j}}=\varepsilon_{j} /\left|\varepsilon_{j}\right|$. The mass eigenvalues are

\footnotetext{
${ }^{3}$ In the usual discussion the $2 \times 2$ mass matrix [see Eq. (14)] has 0 for the upper left corner and the lower right corner given by $m_{R} \neq 0$ but $\ll v$ [24]. Since most of the signatures for pseudoDirac neutrino involve detecting mass splittings one cannot distinguish this from our scenario.
} 


$$
\begin{aligned}
& m_{i}^{+2}=m_{i}^{2}+m_{i}\left|\varepsilon_{i}\right|, \\
& m_{i}^{-2}=m_{i}^{2}-m_{i}\left|\varepsilon_{i}\right|,
\end{aligned}
$$

with $i=1,2,3$. Clearly, $U$ will not diagonalize $\mathbf{m}_{\mathbf{L}}$ in general and also $\mathbf{m}_{\mathbf{D}}$ is diagonalized by $U^{\dagger} \mathbf{m}_{\mathbf{D}} U_{R}$ as for SM charged leptons. We label the mass eigenstates $\nu_{j}^{ \pm}$ corresponding to the eigenvalues of Eq. (21) and as a result the three active neutrinos are related to the mass eigenstates via

$$
\nu_{\alpha L}=\frac{1}{\sqrt{2}} \sum_{j} U_{\alpha j}\left(\nu_{j}^{+}+i \nu_{j}^{-}\right) .
$$

As we have argued before the largest element in $\mathbf{m}_{\mathbf{L}}$ is the $\tau \tau$ component; thus, with the current neutrino oscillation data given by [20] we predict

$$
\varepsilon_{1}: \varepsilon_{2}: \varepsilon_{3} \simeq\left|U_{1 \tau}\right|^{2}:\left|U_{2 \tau}\right|^{2}:\left|U_{3 \tau}\right|^{2} \simeq 0.04: 1: 1
$$

The neutrinos flavor conversion probability can be expressed as

$$
P\left(\nu_{\alpha} \rightarrow \nu_{\beta}\right)=\frac{1}{4}\left|\sum_{j=1}^{3} U_{\alpha j}\left\{e^{-i\left(m_{j}^{+2}\right) \frac{L}{2 E}}+e^{-i\left(m_{j}^{-2}\right) \frac{L}{2 E}}\right\} U_{\beta j}^{*}\right|^{2},
$$

where $L$ is the baseline of the neutrino experiment and $E$ is the neutrino energy. The $\nu_{\alpha}$ survival probability is then

$$
\begin{aligned}
P\left(\nu_{\alpha} \rightarrow \nu_{\alpha}\right)= & \sum_{j=1}^{3}\left|U_{\alpha j}\right|^{4} \cos ^{2} m_{j} \varepsilon_{j} x \\
& +2 \sum_{i>j, 1}^{3}\left|U_{\alpha i}\right|^{2}\left|U_{\alpha j}\right|^{2} \cos \left(m_{i} \varepsilon_{i} x\right) \\
& \times \cos \left(m_{j} \varepsilon_{j} x\right) \cos \left[\left(m_{i}^{+2}-m_{j}^{+2}\right) x\right],
\end{aligned}
$$

where $x=L /(2 E)$ and $m_{i}^{+2}$ is given in Eq. (21). In the limit all $\varepsilon \rightarrow 0$ this reduces to the standard expressions.

Equation (25) shows that there are long wavelength oscillations superimposed on the observed ones. In order to be able to observe the effects of $\varepsilon$, the oscillation length is given by

$$
\ell=125\left(\frac{E}{\mathrm{MeV}}\right)\left(\frac{10^{-5} \mathrm{eV}^{2}}{m \varepsilon}\right) \mathrm{km} .
$$

To get a better grasp of the allowed $\varepsilon$ values given by the current oscillation data, we take as a benchmark the normal neutrino mass hierarchy and assume that the lightness $m_{1} \simeq 0$. Thus, the solar neutrinos and atmospheric neutrino oscillations give $m_{2} \simeq 0.009 \mathrm{eV}$ and $m_{3} \simeq 0.05 \mathrm{eV}$. Solar neutrinos with a base line of $1.5 \times 10^{8} \mathrm{~km}$ will be the most sensitive probe of $\varepsilon$. For our benchmark case, an examination of Eq. (25) shows that the most important contribution is $m_{2} \varepsilon_{2}$. Equation (26) yields $\varepsilon_{2} \lesssim 8 \times 10^{-9} \mathrm{eV}$, which is consistent with the result found in [24]. Using Eq. (12) and the oscillation data, our benchmark model leads to $\varepsilon_{2} \simeq 3.5 \times 10^{-7} \mathrm{eV}$ for $M_{S}=1 \mathrm{TeV}$ and $\varepsilon \simeq 3.5 \times$ $10^{-9} \mathrm{eV}$ for $M_{S}=10 \mathrm{TeV}$. The solar neutrino data favors a heavier scalar in this simple model.

From Eq. (25), it is easy to see that atmospheric neutrinos will be sensitive to $\varepsilon_{3}$. As an estimate, we take the base line to be $10^{4} \mathrm{~km}$ and $\left\langle E_{\nu}^{\mathrm{atm}}\right\rangle \simeq 10 \mathrm{GeV}$ and obtain that $\varepsilon_{3} \lesssim 0.02 \mathrm{eV}$. This is to be compared with our model that gives $\varepsilon_{3} \simeq \varepsilon_{2} \sim 10^{-7}\left(10^{-9}\right) \mathrm{eV}$ [see Eq. (23)] from the solar neutrino data. We note that our assumption of Eq. (12) relies on universal Yukawa couplings, which is unlikely to be exact. For phenomenological purposes, it is best to treat $\varepsilon_{i}$ 's as free parameters to be determined by experiments.

With this in mind we see that the next generation reactor experiment JUNO [28] with a base line of $57 \mathrm{~km}$ and neutrino energy in the $\mathrm{MeV}$ range is well suited for studying pseudoDirac neutrino oscillations with splittings $O\left(10^{-4}\right) \mathrm{eV}$. Smaller $\varepsilon$ will require astrophysical neutrinos sources and neutrino telescopes. We defer a detail study of this intricate oscillation phenomena to a future study. For some early discussions of the pseudo-Dirac neutrino phenomenology, see $[24,29]$. For more recent works see [30-32].

In conclusion by assuming the short range interactions to be dominated, we have broken the connection between $0 \nu \beta \beta$ and direct neutrino mass measurements using kinematics of weak decays of nuclei such as the Katrin experiment [33] and Project 8 [34]. This is not surprising since the neutrino exchange is no longer assumed. If future experiments do not confirm the expected connections within expected uncertainties, then short range interactions must be taken into account. Interestingly, our study has opened up a new connection between $0 \nu \beta \beta$ and the phenomenology of pseudo(quasi)-Dirac neutrinos. These effects may be probed in future neutrino oscillation experiments and neutrino telescopes and further studies are warranted.

\section{HIGH ENERGY COLLIDER PROBES}

To implement the short range dominance in $0 \nu \beta \beta$, we have introduced a moderate number of new scalars. There are a pair of neutral spin- 0 states, $\psi^{0}$ and $t^{0}$. The real parts of which are two Higgs scalars with masses in the Tev range. There is also one heavy pseudoscalar from a linear combination of the imaginary parts. The orthogonal combination will be a massless Majoron since lepton number is spontaneously violated in the model. This can serve as a candidate for dark radiation and the phenomenology has been extensively discussed in the literature [35-37]. In addition, there are two pairs of singly charged scalars $\left(t^{ \pm}, \psi^{ \pm}\right)$and two pairs of doubly charged scalars $\left(\Phi^{ \pm \pm}, \psi^{ \pm \pm}\right)$and a triply charged pair $\psi^{ \pm \pm \pm}$. Their masses 
are all expected to be in the $\mathrm{TeV}$ range. Of all these the experimentally more spectacular ones are the multiply charged states. They are easily produced with sufficient energy. Their production cross sections are enhanced due to the high charges. The LHC search efforts concentrate mainly on the doubly charged ones using multileptons as signatures which we have already discussed. The triply charged states are more unusual can also be searched for at the LHC [16]. Much of the detail phenomenology is model dependent and in particular is sensitive to the parameters of the scalar potential. Instead we will focus on more model independent signatures without having to spell out the details of the potential.

A well-known general mechanism for the pair production of new particles is via the Drell-Yan process. Specifically, we can have

$$
q+\bar{q} \rightarrow \gamma^{*} \rightarrow \psi^{+++} \psi^{---} .
$$

The decays of $\psi$ proceed as

$$
\begin{aligned}
\psi^{+++} \rightarrow W^{+}+ & S_{1}^{++} \\
\downarrow & \\
& \ell^{+}+\ell^{\prime+} .
\end{aligned}
$$

The final signature is a resonance of a same sign dilepton with a same sign $W$-boson. In this reaction all the couplings are known with the only model dependence coming in the branching ratio of $S_{1} \rightarrow \ell \ell^{\prime}$. An equally interesting reaction is

$$
u+\bar{d} \rightarrow W^{+*} \rightarrow \psi^{+++}+S_{1}^{--},
$$

followed by the decay of $\psi^{+++}$as in Eq. (28) and a same opposite sign dilepton recoiling against it. Notice that none of the new charged states couples directly to quarks; hence, the Drell-Yan mechanism is the best for their production.

High energy lepton colliders will be ideal probes for the new states, in particular if we have a $e^{-} e^{-}$collider option. Such an advanced lepton collider is expected to operate with the center of mass $(\mathrm{cm})$ energy in the multi-TeV range [38] and an exploratory luminosity of at least $10^{36} \mathrm{~cm}^{-2} \mathrm{~s}^{-1}$. Optimistically, one can search for direct production of the doubly charged states as a dilepton resonance via

$$
e^{-} e^{-} \rightarrow S_{1}^{--} \rightarrow \ell^{-} \ell^{\prime-},
$$

where $\ell, \ell^{\prime}=e, \mu, \tau$. Since $\bar{y}_{\Phi} \leq 1$, this rate is not be suppressed. If $M_{1}<\sqrt{s}$ with $s$ the cm energy, one will see a peak in the total cross section. For $M_{1}>\sqrt{s}$, the cross section $e^{-} e^{-} \rightarrow S^{*--} \rightarrow \ell^{-} \ell^{\prime-}$ is

$$
\sigma=\frac{y_{e e}^{2} y_{\ell \ell^{\prime}}^{2}}{32 \pi} \frac{s}{\left(s-M_{1}^{2}\right)^{2}} .
$$

For the diagonal terms $\ell=\ell^{\prime}$, it is a factor of 2 larger. This cross section is $O(40) \mathrm{fb}$ for $\sqrt{s}=1 \mathrm{TeV}$ if we set $y_{e e}=y_{\ell \ell^{\prime}}=e$.

Similarly, one can consider the case of $e^{-} e^{-} \rightarrow S^{*--} \rightarrow$ $W^{-} W^{-}$, which will be easier to search for in a lepton collider than a hadron collider. The reaction $e^{-} e^{-} \rightarrow$ $W^{-} W^{-}$is the inverse of $0 \nu \beta \beta$ if the latter proceeds via a virtual $\mathrm{W}$ exchange. Thus, it provides a model independent test of the two-W-boson mechanism for $0 \nu \beta \beta$. Here, the W-boson pair is on shell. This can be seen in the righthand diagram of Fig. 1. Now the two electrons are incoming from the top and the two $\mathrm{W}$-bosons are outgoing and decay into two jets each or $\ell \nu$ pair. This reaction must occur if $0 \nu \beta \beta$ were observed and proceeds via the $2-\mathrm{W}$ exchange. We refer this as inverse $0 \nu \beta \beta$ and was first discussed in [39]. In our model, this is a s-channel process, hence any one of the W-bosons will have an isotropic scattering angle distribution. This contrasts with the previous discussions on this reaction which were mainly focussed on of probing heavy Majorana neutrino exchanges [39]. The latter has a characteristic $t$ channel angular distribution that peaks at $\pi / 2$. It is instructive to note that for a $1 \mathrm{TeV}$ Majorana neutrino $N$ that mixes with $\nu_{e}$ with the mixing parameter $10^{-3}$, the cross section at high $s$ is $\sim 4.2 \mathrm{fb}$. In passing we also note that similar probe reaction at hadron colliders such as the LHC using two $\mathrm{W}$ fusion to two same sign leptons have challenging backgrounds, see, e.g., [40]. On the positive side searches at hadron colliders probe two units lepton number violation that are not both electrons. Up till now the only constraints come from rare meson decays such as $K \rightarrow \pi \mu \mu(\mu e)$ and $\mu^{-} \rightarrow e^{+}$conversion in nuclei.

\section{CONCLUSIONS}

Lepton number violation (LNV) is a crucial question in particle physics. It is intimately connected to the question of the neutrino mass generation, which remains unknown despite tremendous progress in the experimental front in establishing neutrino oscillations. It is also widely believe that it is violated by a small amount in the SM in terms of the small active neutrino Majorana mass. However, this is far from being established. Observation of $0 \nu \beta \beta$ will then be the explicit demonstration of LNV independent of any model. There is now a world wide effort in improving the current experiment [41]. The usual theoretical discussion begins with the assumption of long range exchanges of light Majorana neutrinos as the dominant mechanism for $0 \nu \beta \beta$. Here, we make the assumption that $0 \nu \beta \beta$ proceeds mainly through short range interactions involving the twoW-boson exchange. We also confine ourself to no new gauge interactions for the SM fermions. This turns out to be very restrictive and new scalars with high $S U(2)$ representations can induce such decays. The new physics also generates a small Majorana neutrino mass for $\nu_{e}$ that is insignificant for $0 \nu \beta \beta$. While this is consistent with our 
hypothesis for $0 \nu \beta \beta$ but inconsistent with the oscillation data. We propose to solve it by assuming that the light neutrinos have predominantly Dirac masses and the small Majorana masses induced by the new scalars render them quasi-Dirac particles. The splitting although small but may be detectable in the next generation of neutrino oscillation experiments and/or neutrino telescope. This is a new connection between $0 \nu \beta \beta$ and neutrino physics which is yet to be studied in detail. Conversely the search for evidence of pseudo-Dirac nature of neutrinos can shed light on the mechanism for $0 \nu \beta \beta \mathrm{s}$.
We also noted the new physics signals such as the high charged states that can be explored in hadron colliders. In particular, we find that a high energy $e^{-} e^{-}$will be very useful in testing the origin of LNV and complements the $0 \nu \beta \beta$ studies.

\section{ACKNOWLEDGMENTS}

This work was supported in part by National Center for Theoretical Sciences, Taiwan and Ministry of Science and Technology,Taiwan under MoST-107-2119-M-007013-MY3.
[1] M. J. Dolinski, A. W. P. Poon, and W. Rodejohann, Annu. Rev. Nucl. Part. Sci. 69, 219 (2019).

[2] S. M. Bilenky and C. Giunti, Int. J. Mod. Phys. A 30, 1530001 (2015).

[3] L. Graf, F. E. Deppisch, F. Iachello, and J. Kotila, Phys. Rev. D 98, 095023 (2018).

[4] V. Cirigliano, W. Dekens, J. de Vries, M. L. Graesser, and E. Mereghetti, J. High Energy Phys. 12 (2018) 097.

[5] H. Päs, M. Hirsch, H. V. Klapdor-Kleingrothaus, and S. Kovalenko, Phys. Lett. B 453, 194 (1999).

[6] H. Päs, M. Hirsch, H. V. Klapdor-Kleingrothaus, and S. Kovalenko, Phys. Lett. B 498, 35 (2001).

[7] F. del Aguila, A. Aparici, S. Bhattacharya, A. Santamaria, and J. Wudka, J. High Energy Phys. 06 (2012) 146.

[8] V. Tello, M. Nemevsek, F. ti, G. Senjanovic, and F. Vissani, Phys. Rev. Lett. 106, 151801 (2011).

[9] A. Faessler, S. Kovalenko, and F. Semikov, Phys. Rev. D 58, 055004 (1998).

[10] J. Schechter and J. W. F. Valle, Phys. Rev. D 25, 774 (1982).

[11] M. Duerr, M. Lindner, and A. Merle, J. High Energy Phys. 06 (2011) 091.

[12] C. S. Chen, C. Q. Geng, and J. N. Ng, Phys. Rev. D 75, 053004 (2007).

[13] C. S. Chen, C. Q. Geng, J. N. Ng, and J. M. S. Wu, J. High Energy Phys. 08 (2007) 022.

[14] M. Aaboud et al. (ATLAS Collaboration), Eur. Phys. J. C 78, 199 (2018).

[15] CMS Collaboration, Report No. CMS-PAS-HIG-16-036, 2017.

[16] C. S. Chen, C. Q. Geng, D. Huang, and T. H. Tsai, Phys. Rev. D 87, 077702 (2013).

[17] A. Gando et al. (KamLAND-Zen Collaboration), Phys. Rev. Lett. 110, 062502 (2013).

[18] D. Q. Adams et al. (CUORE Collaboration), Phys. Rev. Lett. 124, 122501 (2020).

[19] A. Smolnikov et al. (GERDA Collaboration), AIP Conf. Proc. 2165, 020024 (2019).
[20] M. Tanabaski et al. (Particle Data Group), Phys. Rev. D 98, 030001 (2018).

[21] W. F. Chang, J. N. Ng, and J. M. S. Wu, Phys. Rev. D 80, 113013 (2009).

[22] L. Wolfenstein, Nucl. Phys. B186, 147 (1981).

[23] S. Petcov, Phys. Lett. 110B, 245 (1982).

[24] A. de Gouvêa, W. C. Huang, and J. Jenkins, Phys. Rev. D 80, 073007 (2009).

[25] M. Kobayashi and C.S. Lim, Phys. Rev. D 64, 013003 (2001).

[26] B. Pontecorvo, Sov. Phys. JETP 6, 429 (1957); ibid. 7, 172 (1958).

[27] Z. Maki, M. Nakagawa, and S. Sakata, Prog. Theor. Phys. 28, 870 (1962).

[28] F. An et al. (JUNO Collaboration), J. Phys. G 43, 030401 (2016).

[29] S. Pakvasa, Nucl. Phys. B, Proc. Suppl. 137, 295 (2004).

[30] G. Anamiati, R. M. fonseca, and M. Hirsch, Phys. Rev. D 97, 095008 (2018).

[31] G. Anamiati, V. de Romeri, M. Hirsch, C. A. Termes, and M. Tórtla, Phys. Rev. D 100, 035032 (2019).

[32] A. Esmaili, Phys. Rev. D 81, 013006 (2010).

[33] M. Aker et al. (KATRIN Collaboration), Phys. Rev. Lett. 123, 221802 (2019).

[34] B. Monreal and J. A. Fromaggio, Phys. Rev. D 80, 051301 (2009).

[35] W. F. Chang, J. N. Ng, and J. M. S. Wu, Phys. Lett. B 730, 347 (2014).

[36] W. F. Chang and J. N. Ng, Phys. Rev. D 90, 065034 (2014).

[37] W. F. Chang and J. N. Ng, J. Cosmol. Astropart. Phys. 07 (2016) 027.

[38] E. Adli et al. (ALEGRO Collaboration), arXiv:1901.10370.

[39] D. London, G. Bélanger, and J. N. Ng, Phys. Lett. B 188, 155 (1987).

[40] J. N. Ng, A. de la Puente, and B. W.-P. Pan, J. High Energy Phys. 12 (2015) 172.

[41] A. Giuliani, arXiv:1910.04688. 